\title{
Acrokeratosis Can Be a Warning Sign of an Underlying Malignancy
}

\author{
Sara Nguyen, Martine Grosber, Jan Gutermuth \\ Department of Dermatology, Universitair Ziekenhuis Brussel, Vrije Universiteit Brussel (VUB), Brussels, Belgium
}

Doi: 10.12890/2019_001108 - European Journal of Case Reports in Internal Medicine - @ EFIM 2019

Received: 01/04/2019

Accepted: $17 / 04 / 2019$

Published: $10 / 05 / 2019$

How to cite this article: Nguyen S, Grosber M, Gutermuth J. Acrokeratosis can be a warning sign of an underlying malignancy. EJCRIM 2019;6: doi:10.12890/2019_001108.

Conflicts of Interests: The Authors declare that there are no competing interests.

Acknowledgements: We want to thank Dr. Ingrid De Pauw for referring this patient to our clinic.

This article is licensed under a Commons Attribution Non-Commercial 4.0 License

\section{ABSTRACT}

Introduction: Some cutaneous manifestations can be the first presentation of an underlying malignancy. These so-called paraneoplastic syndromes can sometimes be very subtle or strongly resemble other benign cutaneous diseases.

Aims: In this report, we want to emphasize the need for further investigation of eczema-like cutaneous presentations which develop at a later age and are recalcitrant to therapy. Exclusion of an underlying malignancy needs to be considered.

Patients and Methods: A 53-year-old man with thickening of the skin, more pronounced on the hands, feet and face, was diagnosed with classic nodular sclerosing Hodgkin disease based on cutaneous presentation.

Results: After two rounds of chemotherapy (adriamycin, bleomycin, vinblastine and dacarbazine), the cutaneous symptoms had disappeared. After six rounds of chemotherapy and 14 months of follow-up, the patient is still in remission without recurrence of the cutaneous symptoms. Conclusions: Cutaneous symptoms recalcitrant to adequate treatment should raise suspicion and prompt further investigation to exclude an underlying malignancy. A multidisciplinary approach with the dermatology department can accelerate diagnosis and improve the patient's prognosis.

\section{LEARNING POINTS}

- Skin diseases can reflect internal manifestations or diseases.

- Vigilance, timely recognition and a multidisciplinary approach are important in a patient with atypical cutaneous manifestations.

- A paraneoplastic skin syndrome should be considered in persistent skin disease not responding to treatment and starting in middle-aged patients with B symptoms.

\section{KEYWORDS}

Acrokeratosis paraneoplastica, Bazex syndrome, paraneoplastic syndrome

\section{CASE DESCRIPTION}

A 53-year-old patient attended our clinic because of recalcitrant eczema (Figs. 1-3). His symptoms had started 6 months previously with the development of thickened, dry and itchy skin, especially on the face, palms of the hand and soles of the feet. He had been treated with a topical steroid but showed no improvement. Systemic treatment with methylprednisolone was then started and improved his cutaneous symptoms. However, the skin findings reappeared as soon as this treatment was stopped.

On presentation at our clinic, the patient reported no history of atopy. He had lost $10 \mathrm{~kg}$ in the previous months because of loss of appetite and diarrhoea that had started 3 weeks prior to his consultation. He was an ex-smoker but did not have cough or dyspnoea. He did not have fever. 


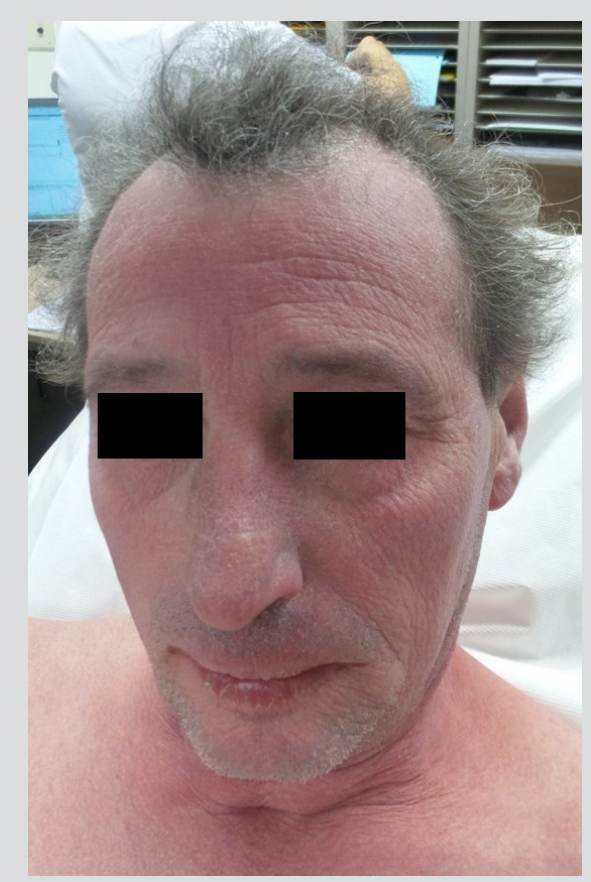

Figure 1. Thickened, dry and erythematous skin on the face of the patient

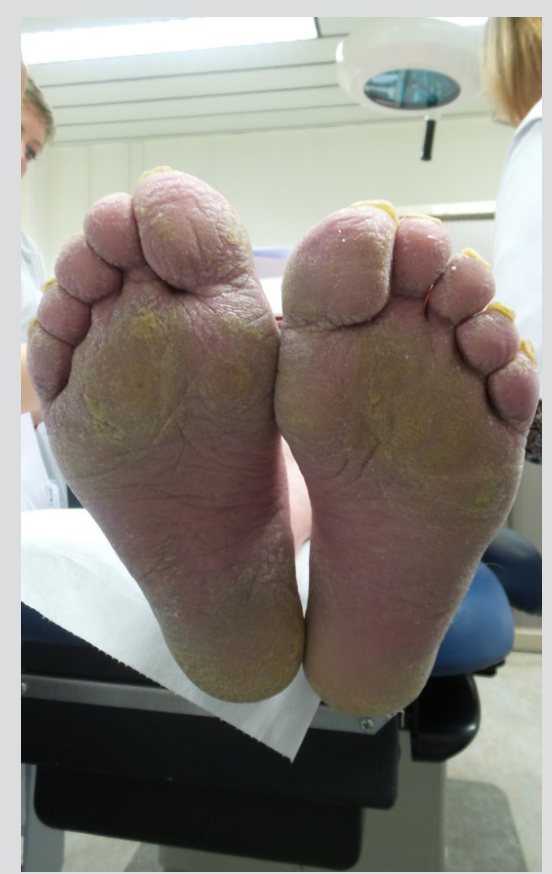

Figure 2. Thickened, dry and erythematous skin on the soles of the patient's feet

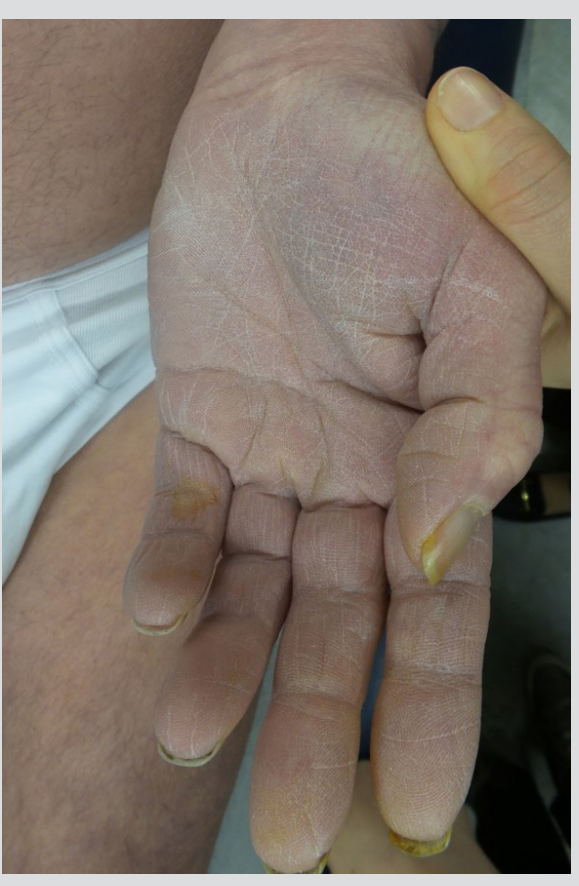

Figure 3. Thickened, dry and erythematous skin on the palms of the patient's hands

A skin biopsy was performed, which showed no disease-specific findings. A blood sample revealed an elevated C-reactive protein and erythrocyte sedimentation rate. Because of the diarrhoea and weight loss, we performed a colonoscopy, which was normal. A CT scan of the thorax and abdomen revealed multiple retroperitoneal lymphadenopathies (Fig. 4).

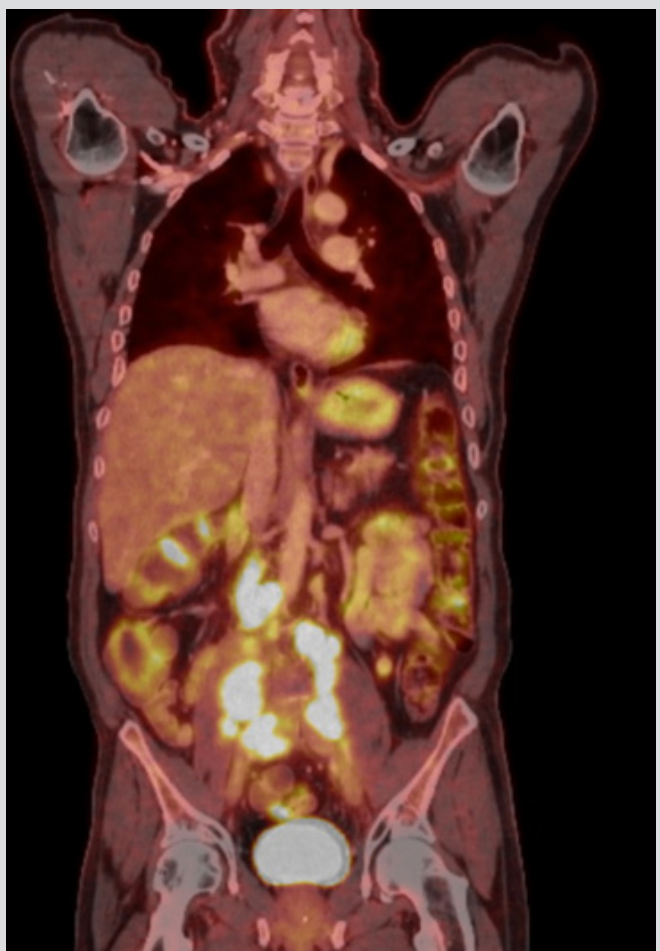

Figure 4. Retroperitoneal lymph adenopathies on a PET-CT scan 
A punction of these lymphadenopathies was performed and confirmed the diagnosis of classic nodular sclerosing Hodgkin disease. The skin symptoms were attributed to a paraneoplastic skin syndrome called acrokeratosis paraneoplastica. The patient was treated in our haematology department where chemotherapy (adriamycin, bleomycin, vinblastine and dacarbazine) was started. After two rounds of chemotherapy, the skin symptoms had disappeared (Figs. 5 and 6). There was no recurrence of skin symptoms after completion of six rounds of chemotherapy. The patient is still in full remission 1 year later.
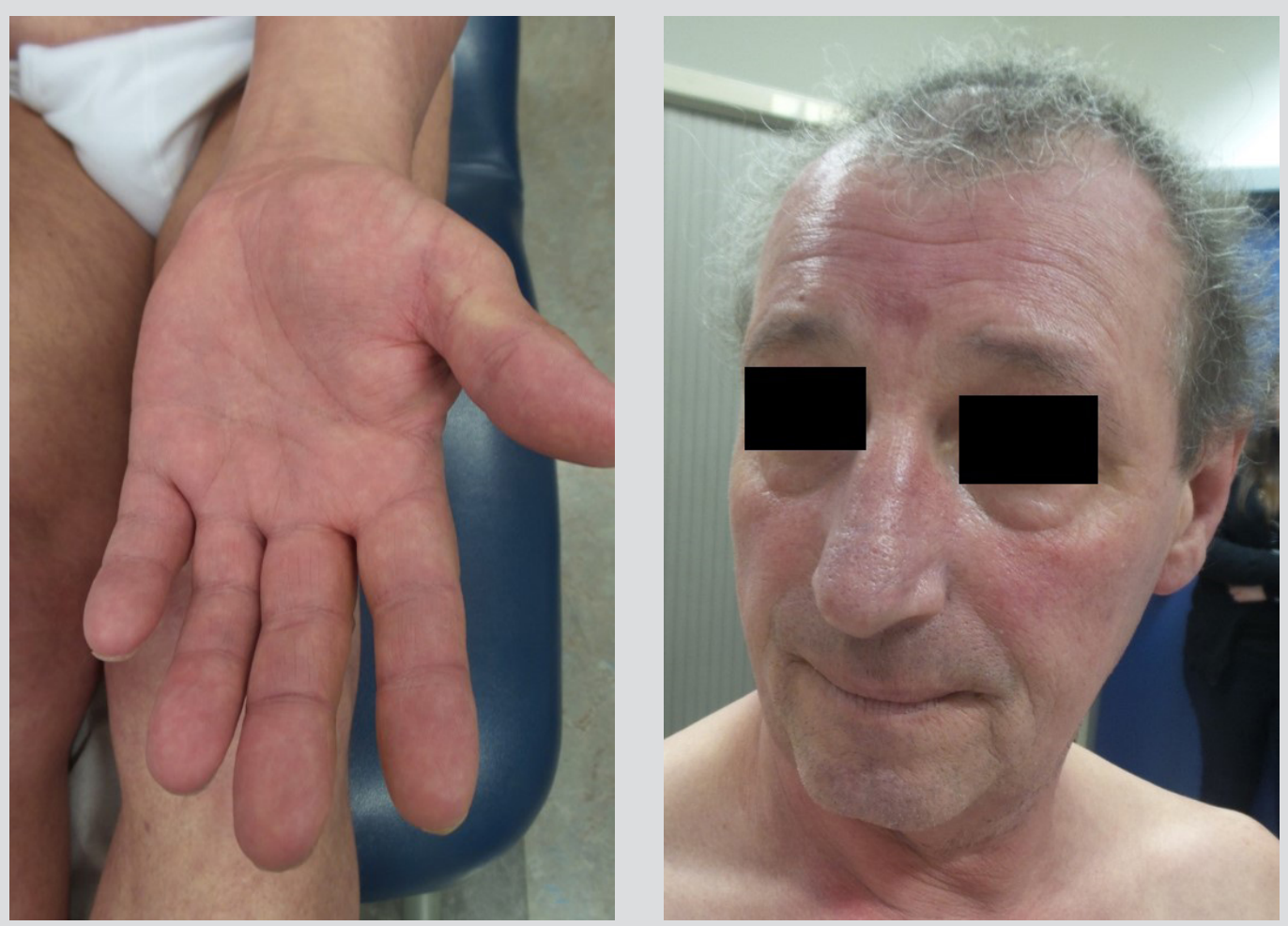

Figure 5. Absolute clearance of paraneoplastic skin symptoms on the patient's hands after chemotherapy

Figure 6. Absolute clearance of paraneoplastic skin symptoms on the patient's face after chemotherapy

\section{DISCUSSION}

Acrokeratosis paraneoplastica (AP), also called Bazex syndrome, is a rare cutaneous paraneoplastic disease that is linked to a variety of internal malignancies. However, it is mostly associated with squamous cell carcinomas of the aerodigestive tract or cervical or mediastinal lymph node metastases ${ }^{[1]}$. Other associated neoplasms include colon adenocarcinoma, gastric adenocarcinoma, Hodgkin disease, T-cell lymphoma, small cell lung carcinoma, lung adenocarcinoma, hepatocarcinoma, multiple myeloma, thymoma, cutaneous squamous cell carcinoma, prostate adenocarcinoma, and vulvar, uterine and bladder carcinoma ${ }^{[2]}$. AP generally affects middle-aged white men. The cutaneous eruption precedes the diagnosis of an underlying malignancy in $67 \%$ of cases. The reported median interval between the onset of the skin changes and the diagnosis of the neoplasia is as long as 1 year. In $15 \%$ of cases, the skin symptoms appear after diagnosis of the malignancy. In the other patients, the diagnosis of the neoplasia and the onset of the skin lesions occur at the same time ${ }^{[3]}$. Skin symptoms are characterized by a hyperkeratotic, psoriasiform or eczematiform eruption with an acral distribution but may also involve the nose and ears ${ }^{[4]}$. In dark-skinned patients, hyperpigmentation is the predominant skin finding ${ }^{[2]}$.

The diagnosis of AP can be a challenge because of its resemblance to other more common cutaneous diseases such as treatment-resistant eczema, psoriasis and superficial fungal infections. The differential diagnosis also includes hereditary forms of palmoplantar keratoderma, such as Howel-Evans syndrome. However, skin manifestations in palmoplantar keratoderma start in childhood, do not disappear after chemotherapy, and can also affect other family members ${ }^{[5]}$.

In AP, paraneoplastic skin findings will disappear after effective treatment of the underlying tumour. A relapse of the skin manifestations often indicates progression of the malignancy ${ }^{[1]}$.

In conclusion, the development of recalcitrant hyperkeratotic skin manifestations affecting the ears, nose, hands and feet and not responding to adequate therapy, and associated with B symptoms, should raise suspicion of a paraneoplastic syndrome. 


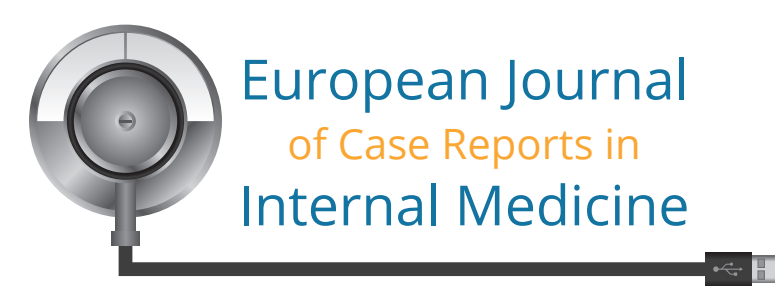

Complete evaluation of the upper aerodigestive tract is then mandatory. If negative, screening for other malignancies reported to occur with AP should be performed.

It is important that internal medicine doctors be aware of the association between skin symptoms and internal malignancy since a delay in diagnosis could result in a worse prognosis.

\section{REFERENCES}

1. Räßler F, Goetze S, Elsner P. Acrokeratosis paraneoplastica (Bazex syndrome) - a systematic review on risk factors, diagnosis, prognosis and management. J Eur Acad Dermatol Venereol 2017;31:119-1136.

2. Valdivielso M, Longo I, Suárez R, Huerta M, Lázaro P. Acrokeratosis paraneoplastica: Bazex syndrome. J Eur Acad Dermatol Venereol 2005;19:340-344.

3. Rao R, Shenoi SD. Acrokeratosis paraneoplastica (Bazex syndrome): an atypical presentation. Dermatol Online J 2004;10:21.

4. McClatchey TM, Haynes D, Korcheva VB, Keller J. Acrokeratosis paraneoplastica (Bazex syndrome) associated with peripheral T-cell lymphoma. JAAD Case Rep 2018;5:86-88.

5. Sroa N, Witman P. Howel-Evans syndrome: a variant of ectodermal dysplasia. Cutis 2010; : 183-185. 